\title{
Comment on "Structural Determinants of Drug Partitioning in Surrogates of Phosphatidylcholine Bilayer Strata"
}

\author{
William E. Acree, Jr., ${ }^{* \dagger}$ Michela Brumfield, ${ }^{\dagger}$ and Michael H. Abraham ${ }^{\ddagger}$ \\ ${ }^{\dagger}$ Department of Chemistry, University of North Texas, 1155 Union Circle Drive \#305070, Denton, Texas 76203, United States \\ ${ }^{\ddagger}$ Department of Chemistry, University College London, 20 Gordon Street, London WC1H OAJ, U.K.
}

Mol. Pharmaceutics, 2013, 10 (10), 3684-3696. DOI: 10.1021/mp400204y

ABSTRACT: The Abraham model correlation for describing the partitioning behavior of solutes between water and hexadecane was redetermined using the measured partition coefficient data and solute descriptors derived from experimental data. The newly derived correlation provides a much better mathematical description of the observed partition coefficient data than the correlation given in the published paper.

KEYWORDS: phosphatidylcholine, partition coefficients, equilibrium, Abraham model

T $\mathrm{n}$ a recent article appearing in this Journal, Lukacova et al. ${ }^{1}$ experimentally determined the partition coefficients of 113 selected molecular compounds between hexadecane and diacetyl phosphatidylcholine (DAcPC) by measuring the equilibrium solute concentrations using ultraviolet spectroscopy or gas chromatographic/mass spectrometry methods. As part of the study, the authors correlated the logarithms of the measured partition coefficients, $\log P_{\mathrm{C} 16 / \mathrm{DAcPC}}$, with the Abraham general solvation parameter model:

$$
\log P=c_{\mathrm{p}}+e_{\mathrm{p}} \cdot \mathbf{E}+s_{\mathrm{p}} \cdot \mathbf{S}+a_{\mathrm{p}} \cdot \mathbf{A}+b_{\mathrm{p}} \cdot \mathbf{B}+v_{\mathrm{p}} \cdot \mathbf{V}
$$

The independent variables in eq 1 are descriptors of the solutes. In brief, $\mathbf{E}$ is the solute excess molar refractivity in units of $\left(\mathrm{cm}^{3}\right.$ $\left.\mathrm{mol}^{-1}\right) / 10, \mathbf{S}$ is the solute dipolarity/polarizability, $\mathbf{A}$ and $\mathbf{B}$ are the overall or summation hydrogen-bond acidity and basicity, and $\mathbf{V}$ is the McGowan volume in units of $\left(\mathrm{cm}^{3} \mathrm{~mol}^{-1}\right) / 100$. The equation coefficients $\left(c_{\mathrm{p}}, e_{\mathrm{p}}, s_{\mathrm{p}}, a_{\mathrm{p}}, b_{\mathrm{p}}\right.$, and $\left.v_{\mathrm{p}}\right)$ are not just fitting constants but reflect the chemistry of the system in question. In particular, the a-coefficient will reflect the hydrogen-bond basicity of the system (because a hydrogenbond solute acid will interact with a system that is a hydrogenbond base), and the $b$-coefficient will reflect the hydrogen-bond acidity of the system. Numerical values of the equation coefficients are obtained by multilinear regression analysis and serve to characterize the partitioning system under consideration.

The derived Abraham correlation for $\log P_{\mathrm{C} 16 / \mathrm{DAcPC}}$ was compared to the correlations obtained by the authors for the water-to-hexadecane partition coefficient, $\log P_{\mathrm{C} 16 / \mathrm{W}}$

$$
\begin{aligned}
& \log P_{\mathrm{C} 16 / \mathrm{W}}=0.342(0.201)+0.885(0.202) \mathbf{E} \\
& \quad-1.982(0.245) \mathbf{S}-3.300(0.242) \mathbf{A}-4.568(0.298) \mathbf{B} \\
& +4.197(0.232) \mathbf{V} \quad\left(\mathrm{SD}=0.412, r^{2}=0.971, F=489\right)
\end{aligned}
$$

and for the correlation water-to-1-octanol partition coefficient, $\log P_{\mathrm{O} / \mathrm{w}}$. The water-to-hexadecane partition coefficient correlation appears to have been derived by the authors from available experimental $\log P$ data given in Table $S 1$ of the Supporting Information, while the water-to-1-octanol partition coefficient expression (and its associated statistics) is quite close to the correlation published by Abraham and co-workers; ${ }^{2}$ note that Abraham and co-workers used the alternative hydrogen bond basicity descriptor $\mathbf{B}^{\mathbf{o}}$ for the water-to-1-octanol partition coefficient. Footnote "a" at the bottom of Table 3 states that only compounds with experimental solvatochromic parameters $(N=78)$ were used in determining the $\log P$ equations, and its placement in the column heading implies that the footnote pertains to all correlations given the table. The statistical information associated with eq 2 includes the standard deviation, SD, squared correlation coefficient, $r^{2}$, and the Fisher F-factor, F.

In the published paper the authors stated that the coefficients for the C16/W system were in good agreement with published data given by Abraham and co-workers. ${ }^{2}$ The correlation reported by Abraham and co-workers for the water-tohexadecane partition coefficient was:

$$
\begin{aligned}
& \log P_{\mathrm{C} 16 / \mathrm{W}}=0.087+0.667 \mathbf{E}-1.617 \mathrm{~S}-3.587 \mathbf{A} \\
& -4.869 \mathbf{B}+4.433 \mathbf{V} \\
& \left(N=370, \mathrm{SD}=0.124, r^{2}=0.996, F=20236\right)
\end{aligned}
$$

Equation 3 is based on 370 data points. The large standard deviation of $\mathrm{SD}=0.412 \mathrm{log}$ units associated with eq 2 above is quite surprising given that the statistical information reported by Abraham and co-workers for $\log P_{\mathrm{C} 16 / \mathrm{W}}$ is much better, $\mathrm{SD}=$ 0.124 versus $S D=0.412 \log$ units.

The purpose of the present commentary is not to criticize the excellent work of Lukacova et al. ${ }^{1}$ but rather to examine why the standard deviation of their $\log P_{\mathrm{C} 16 / \mathrm{W}}$ correlation is so much larger than that reported previously by Abraham and co-

Received: May 22, 2014

Revised: October 2, 2014

Accepted: January 13, 2015

Published: January 13, 2015 
workers on a much larger data set. Lukacova et al. presumably obtained eq 2 by analyzing the experimental $\log P_{\mathrm{C} 16 / \mathrm{W}}$ in Table S1 for those compounds for which "experimental" values of the solute descriptors could be found. This would be the light-blue shaded values in Table S1. The authors provided no literature reference for the $\log P_{\mathrm{C} 16 / \mathrm{W}}$ correlation or data set of $\log P_{\mathrm{C} 16 / \mathrm{W}}$ values and solute descriptors other than that tabulated in Table S1. The equation coefficients differ from those published by Abraham and co-workers. ${ }^{2}$

Our analysis of the experimental data in Table S1 gave a much better Abraham model correlation:

$$
\begin{aligned}
& \log P_{\mathrm{C} 16 / \mathrm{W}}=-0.018(0.234)+0.519(0.196) \mathbf{E} \\
& -1.399(0.194) \mathbf{S}-3.522(0.195) \mathbf{A}-4.749(0.235) \mathbf{B} \\
& +4.451(0.311) \mathbf{V} \\
& \left(N=44, S D=0.269, r^{2}=0.984, F=453.7\right)
\end{aligned}
$$

that is more in line with standard deviation expectations. Excluded from our analysis are those compounds for which the solute descriptors were not blue-shaded in Table S1. ${ }^{1}$ The authors may have inadvertently included in the development of eq 2 a few compounds for which "measured" solute descriptors were not known or perhaps the authors included estimated log $P_{\mathrm{C} 16 / \mathrm{W}}$ values. Table $\mathrm{S1}^{1}$ does contain several compounds having solute descriptors estimated by the commercial Absolv software $^{3}$ and having $\log P_{\mathrm{C} 16 / \mathrm{w}}$ estimated by a ClogP-based fragmentation model. ${ }^{4}$

The authors state in the manuscript that "to obtain the most precise coefficient values, only the compounds with the solvatochromic properties determined from experimental values (78 compounds marked in Table S1 in the Supporting Information) were used." We do not think that inclusion of a large number of predicted $\log P_{\mathrm{C} 16 / \mathrm{W}}$ values in the regression analysis would give the most precise coefficient values if the goal is to develop a correlation for describing experimental log $P_{\mathrm{C} 16 / \mathrm{W}}$ values. There are 34 compounds in Table $\mathrm{S} 1$ of ref 1 for which experimentally based solute descriptors were given but not measured $\log P_{\mathrm{C} 16 / \mathrm{W}}$ values. We did find an experimental $\log P_{\mathrm{C} 16 / \mathrm{W}}$ value for one of the 34 compounds, $\log P_{\mathrm{C} 16 / \mathrm{w}}=$ $2.17^{2}$ for $N, N$-dimethylaniline. The experimental value differs from the estimated value given in Table $\mathrm{S} 1$ of ref $1, \log P_{\mathrm{C} 16 / \mathrm{w}}$ $=3.721$, by more than $1.5 \log$ units. The Abraham model does provide a much better mathematical description of the measured $\log P_{\mathrm{C} 16 / \mathrm{W}}$ data than is indicated by the standard deviation of $S D=0.412$ in Table 3 of the Lukacova et al. paper.

During the course of our computations we did note that Lukacova et al. ${ }^{1}$ used the $\mathbf{B}^{\mathbf{o}}$ solute descriptor for aniline, 4aminoacetophenone, and quinoline. The $\mathbf{B}^{\mathbf{o}}$ solute descriptor is intended to be used for those practical partitioning systems where the organic phase contains an appreciable amount of water, such as the wet alcohol/water. ${ }^{5,6}$ We do not think that this is the case for hexadecane/water system. Since water and hexadecane are almost completely immiscible, one should be using the $\mathbf{B}$ solute descriptor for the aniline $(\mathbf{B}=0.410)$, 4aminoacetophenone $(\mathbf{B}=0.650)$, and quinoline $(\mathbf{B}=0.540)$. Reanalysis of the $\log P_{\mathrm{C} 16 / \mathrm{W}}$ using the $\mathbf{B}^{\mathbf{o}}$ solute descriptors should have very little effect on the determined equation coefficients and associated statistics because there are very few compounds in the data set for which the $\mathbf{B}$ and $\mathbf{B}^{\mathbf{o}}$ solute descriptors were different.

\section{AUTHOR INFORMATION}

\section{Corresponding Author}

*E-mail: acree@unt.edu.

\section{Notes}

The authors declare no competing financial interest.

\section{REFERENCES}

(1) Lukacova, V.; Natesan, S.; Peng, M.; Tandlich, R.; Wang, Z.; Lynch, S.; Subramaniam, R.; Balaz, S. Structural determinants of drug partitioning in surrogates of phosphatidylcholine bilayer strata. Mol. Pharmaceutics 2013, 10, 3684-3696.

(2) Abraham, M. H.; Chadha, H. S.; Whiting, G. S.; Mitchell, R. C. Hydrogen bonding. 32. An analysis of water-octanol and water-alkane partitioning and the delta log P parameter of Seiler. J. Pharm. Sci. 1994, $83,1085-1100$.

(3) Absolv, build 2203; Advanced Chemistry Development Inc.: Toronto, Canada, 2013.

(4) Natesan, S.; Wang, Z.; Lukacova, V.; Peng, M.; Subramaniam, R.; Lynch, S.; Balaz, S. Structural determinants of drug partitioning in $\mathrm{n}$ hexadecane/water system. J. Chem. Inf. Model. 2013, 53, 1424-1435.

(5) Abraham, M. H. Scales of solute hydrogen-bonding: Their construction and application to physicochemical and biochemical processes. Chem. Rev. 1993, 22, 73-83.

(6) Abraham, M. H.; Nasezadeh, A.; Acree, W. E., Jr. Correlation and prediction of partition coefficients from the gas phase and from water to alkan-1-ols. Ind. Eng. Chem. Res. 2008, 47, 3990-3995. 